\title{
The Inflammasomes are implicated in both Depression and Coronary Artery disease in Vietnam veterans.
}

\author{
Wole Akosile, MBBS, MPH, FRANZCP, Joanne Voisey, PhD, Bruce Lawford, MD, \\ David Colquhoun, Ross McD Young, PhD
}

School of Psychology and Counselling,

Queensland University of Technology, Kelvin Groove, Brisbane Australia .

\section{Background}

- We hypothesise that there is a syndemic interaction between coronary artery disease and Post-traumatic stress disorder in Veteran populations.

- 2015 Edmonson et al- Those living with PTSD are 1.55 times more likely to have CAD.

- 2018 Akosile et al-Those living with PTSD are 1.61 times more likely to have CAD.

- 2016-Pollard et al-genetic overlap.

- Pollard and colleagues find that 37 of the PTSD candidate risk genes are also candidate independent risk genes for CVD. Three of the five main upstream regulators are from the inflammasome family .

- Inflammasome are critical in initiating and signalling a series of inflammatory activity across several body systems.

- The are innate immune system receptors and sensor.

- They regulate the activation of caspase-1.

- They induce inflammation in response to infectious microbes and molecules derived from host proteins.

- The inflammasomes detect danger signals such as pathogen associated molecular patterns (PAMPs) or damage associated molecular patterns (DAMPs)

- Leading to an aggregation of inflammasome complexes

\section{Aim of studies}

To assess the role played by NLRP12 and NLRP3 polymorphisms in both Depression and raised Coronary calcium scores in Vietnam veterans exposed to trauma.

To assess the link between NLRP3 polymorphisms and coronary artery disease in a well characterised sample of post-Vietnam veterans $(n-299)$

\section{Methods}

Associations of genetic markers with psychological measures

$N L R P 3$ represented by 8 genetic markers $N L R P 12$ represented by 5 genetic markers

\section{Findings}

- The NLRP12 rs34436714 AC genotype is a risk genotype for depression in a Caucasian cohorts. - Also for first time the rs34971363 (CG) and rs6509825 (CT) genotype have been associated with raised coronary calcium score in a Caucasian sample.

- Carriers of NLRP3 rs10159239-A allele (AA+AG) had an increased risk of having a higher coronary calcium score

\section{Conclusion}

There is a possible syndemic interaction between PTSD and CAD in the sub-population of Vietnam veterans mediated by inflammation and inflammasomes

Syndemic Model (PTSD and CAD) [139]

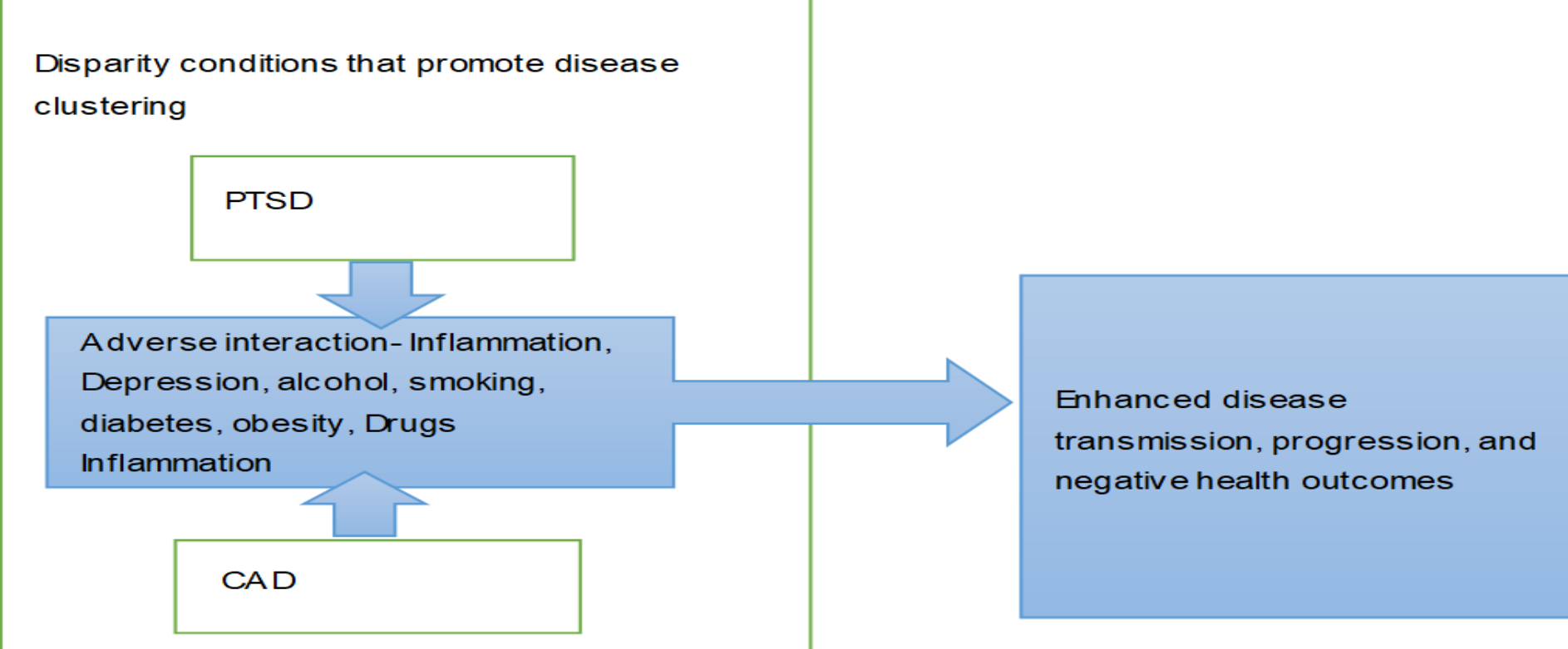

